\title{
Entropy production for a class of inverse SRB measures
}

\author{
Eugen Mihailescu and Mariusz Urbański
}

\begin{abstract}
We study the entropy production for inverse SRB measures for a class of hyperbolic folded repellers presenting both expanding and contracting directions. We prove that for most such maps we obtain strictly negative entropy production of the respective inverse SRB measures. Moreover we provide concrete examples of hyperbolic folded repellers where this happens.
\end{abstract}

Mathematics Subject Classification 2000: 37D20, 37D35, 37A35, 37C70.

Keywords: Inverse SRB measures, folded repellers, Anosov endomorphisms, entropy production.

\section{Introduction.}

In this short note we study certain properties related to the entropy production of invariant measures on a repeller $\Lambda$ for a smooth $\mathcal{C}^{2}$ endomorphism $f: M \rightarrow M$, defined on a Riemannian manifold $M$. By repeller we mean a compact invariant set $\Lambda$ for which there exists a neighbourhood $U$ such that $\bar{U} \subset f(U)$ and such that $\Lambda=\bigcap_{n \leq 0} f^{n}(U)$. Sometimes we will call such repellers, folded repellers to emphasize that the map is not necessarily invertible on them. We shall work in the sequel with hyperbolic saddle-type repellers, i.e $f$ is assumed to be hyperbolic as a non-invertible map on $\Lambda$ (see [16], [9], etc.) and it has both stable and unstable directions in the tangent bundle; the map $f$ is not assumed to be expanding on $\Lambda$. For Anosov diffeomorphisms or for diffeomorphisms having a hyperbolic attractor, we have the existence of Sinai-Ruelle-Bowen (SRB) measures (see for instance [17], [4], [14], [1], [2], [3], [20], etc.) SRB measures exist also for smooth endomorphisms with hyperbolic attractors and are equal to the equilibrium measures of the unstable potentials, on inverse limit spaces (see [12]). In [8] it was introduced also an inverse SRB measure $\mu^{-}$ on a hyperbolic folded saddle repeller $\Lambda$, which may be interpreted as the distribution of past trajectories of Lebesgue almost all points in a neighbourhood of $\Lambda$; this includes also the case of Anosov endomorphisms.

For a non-invertible smooth map $f$ on a Riemannian manifold $M$ and an $f$-invariant probability $\mu$ on $M$, Ruelle defined in [15] the entropy production of $\mu$ by $e_{f}(\mu):=F_{f}(\mu)-\int \log |\operatorname{det}(D f)(x)| d \mu(x)$, where $F_{f}(\mu)$ is the folding entropy of $\mu$ with respect to $f . F_{f}(\mu)$ is defined as the conditional entropy $H_{\mu}\left(\epsilon \mid f^{-1} \epsilon\right)$, where $\epsilon$ is the single point partition. In general it is a natural problem to establish the sign of the entropy production of an invariant measure. 
We are concerned here with finding the sign of the entropy production especially for inverse SRB measures, and to prove that there are many (in a certain sense to be discussed later) examples of repellers whose respective inverse SRB measures have negative entropy production rate. We study first the case of hyperbolic repellers, then the case of Anosov endomorphisms and their associated inverse SRB measures. In particular this applies to hyperbolic toral endomorphisms $f_{A}$, and perturbations of them. Recall that perturbations of hyperbolic toral endomorphisms are not necessarily conjugated to the linear toral endomorphisms ([11]).

Here we will show in Proposition 1 that for hyperbolic saddle-type repellers, the entropy production of the respective inverse SRB measure $\mu^{-}$is less or equal than 0 , and that it is equal to 0 only if $\mu^{-}$is equal to the SRB measure $\mu^{+}$, in which case the Jacobian (in the Euclidean sense) is cohomologous to a constant. Moreover we show that in fact most maps in a neighbourhood of a given Anosov endomorphism $f$, have inverse SRB measures with strictly negative entropy production. Then in Corollary 1 we prove that for most endomorphisms in the sense discussed in Proposition 1, the associated inverse SRB measure is not a limit of measures of type $\frac{1}{n} \sum_{0 \leq k<n} \rho \circ f^{-k}$, where $\rho$ is absolutely continuous with respect to Lebesgue measure.

In Corollary 2 we construct explicit examples of perturbations of hyperbolic toral endomorphisms, whose inverse SRB measures have strictly negative entropy production. Also in the same Corollary we show that there are examples of perturbations of toral endomorphisms whose forward SRB measures have strictly positive entropy production.

Finally in Corollary 3 we study the entropy production also for a hyperbolic folded repeller which is not Anosov.

\section{Entropy production for inverse SRB measures. Examples of repellers.}

Let us consider a $\mathcal{C}^{2}$ non-invertible map $f: M \rightarrow M$ on a compact Riemannian manifold $M$, and $\Lambda$ be a transitive repeller of $f$, such that $f$ is hyperbolic on $\Lambda$. Sometimes $\Lambda$ may be the whole manifold as in the case of Anosov endomorphisms. Hyperbolicity is understood here in the sense of non-invertible maps, i.e the unstable tangent spaces depend on the whole backward trajectories from the inverse limit space $\hat{\Lambda}:=\left\{\hat{x}=\left(x, x_{-1}, x_{-2}, \ldots,\right)\right.$ with $\left.x_{-i} \in \Lambda, f\left(x_{-i}\right)=x_{-i+1}, i \geq 1\right\}$, see [16], [9]. Also the local unstable manifolds depend on backward trajectories; there is some $r>0$ and local stable and local unstable manifolds, $W_{r}^{s}(x)$ and $W_{r}^{u}(\hat{x})$ for any $\hat{x} \in \hat{\Lambda}$. We use a standard notation, namely $D f_{s}(x):=\left.D f\right|_{E_{x}^{s}}, x \in \Lambda$ and $D f_{u}(\hat{x}):=\left.D f\right|_{E_{\hat{x}}^{u}}, \hat{x} \in \hat{\Lambda}$. Examples and properties of endomorphisms with some hyperbolicity have been studied by many authors, for instance [4], [11], [16], [18], [6], [19], [7], [9], etc. Notice that if $\Lambda$ is a repeller, then the local stable manifolds are contained in $\Lambda$. Hyperbolicity on $\Lambda$ assures the existence of a unique equilibrium (Gibbs) measure $\mu_{\phi}$ for any given Hölder continuous potential $\phi$ on $\Lambda$; equilibrium measures are of great interest and have been studied intensively in the literature (for instance [17], [1], [14], [3], [5], [9], etc.)

Let us now recall the notion of inverse SRB measure, introduced in [8]. Let $\Lambda$ be a connected 
hyperbolic repeller for a $\mathcal{C}^{2}$ endomorphism $f: M \rightarrow M$ defined on a Riemannian manifold $M$, and assume $f$ has no critical points in $\Lambda$. We assume that each point in $\Lambda$ has a constant number of $f$-preimages in $\Lambda$; this happens for instance if $\Lambda$ is a connected repeller (see [8]). Let $V$ be a neighbourhood of $\Lambda$ in $M$ and for any $z \in V$ define the measures

$$
\mu_{n}^{z}:=\frac{1}{n} \sum_{y \in f^{-n} z \cap V} \frac{1}{d(f(y)) \ldots d\left(f^{n}(y)\right)} \sum_{i=1}^{n} \delta_{f^{i} y}
$$

where $d(y)$ is the number of $f$-preimages belonging to $V$ of a point $y \in V(d(\cdot)$ is called also the degree function). Then we proved in [8] that there exists an $f$-invariant measure $\mu^{-}$on $\Lambda$, a neighbourhood $V$ of $\Lambda$, a Borel set $A \subset V$ with $m(V \backslash A)=0$ (where $m$ is the Lebesgue measure on $M)$ and a subsequence $n_{k} \rightarrow \infty$ so that for any $z \in A, \mu_{n_{k}}^{z} \underset{k \rightarrow \infty}{\rightarrow} \mu^{-}$. The measure $\mu^{-}$is called the inverse SRB measure on the repeller. It was proved in [8] that $\mu^{-}$is equal to the equilibrium measure of $\Phi^{s}(x):=\log \left|\operatorname{det} D f_{s}(x)\right|, x \in \Lambda$. This inverse SRB measure is not just the SRB measure for $f^{-1}$, since $f$ is non-invertible. From [8] $\mu^{-}$is the unique $f$-invariant measure $\mu$ which has absolutely continuous conditional measures on local stable manifolds; if $\left.f\right|_{\Lambda}$ is $d$-to- 1 , then $\mu^{-}$is also the unique $f$-invariant probability on $\Lambda$ satisfying an inverse Pesin entropy formula:

$$
h_{\mu^{-}}(f)=\log d-\int_{\Lambda} \sum_{i, \lambda_{i}\left(\mu^{-}, x\right)<0} \lambda_{i}\left(\mu^{-}, x\right) m_{i}\left(\mu^{-}, x\right) d \mu^{-}(x),
$$

where $\lambda_{i}\left(\mu^{-}, x\right)$ are the Lyapunov exponents of $\mu^{-}$at $x$, with multiplicity $m_{i}\left(\mu^{-}, x\right)$. At the same time, for an Anosov endomorphism $f$ on $M$ (or for a hyperbolic attractor), we know from [12] that there exists a unique SRB measure $\mu^{+}$which satisfies a Pesin entropy formula, and which is the projection of the equilibrium measure of the unstable potential $\Phi^{u}(\hat{x}):=-\log \left|\operatorname{det} D f_{u}(\hat{x})\right|, \hat{x} \in \hat{M}$. We give now the definition of folding entropy, and entropy production, according to Ruelle [15].

Definition 1. Let $f: M \rightarrow M$ be an endomorphism and $\mu$ an $f$-invariant probability on $M$, then the folding entropy $F_{f}(\mu)$ of $\mu$ is the conditional entropy: $F_{f}(\mu):=H_{\mu}\left(\epsilon \mid f^{-1} \epsilon\right)$, where $\epsilon$ is the partition into single points. Also define the entropy production of $\mu$ by:

$$
e_{f}(\mu):=F_{f}(\mu)-\int \log |\operatorname{det} D f(x)| d \mu(x)
$$

From [13] it follows that we can use the measurable single point partition $\epsilon$ in order to desintegrate the invariant measure $\mu$ into a canonical family of conditional measures $\mu_{x}$ supported on the finite fiber $f^{-1}(x)$ for $\mu$-a.e $x$. Thus the entropy of the conditional measure of $\mu$ on $f^{-1}(x)$ is $H\left(\mu_{x}\right)=-\Sigma_{y \in f^{-1}(x)} \mu_{x}(y) \log \mu_{x}(y)$. From [10] we have also $J_{f}(\mu)(x)=\frac{1}{\mu_{f(x)}(x)}, \mu-$ a.e $x$, hence

$$
F_{f}(\mu)=\int \log J_{f}(\mu)(x) d \mu(x)
$$

The following Proposition gives conditions for Anosov endomorphisms to have inverse SRB measures of negative entropy production, and shows that "many" endomorphisms fall in this category. An important class of Anosov endomorphisms to which the next Proposition applies, are the hyperbolic toral endomorphisms and their perturbations. 
Proposition 1. Let $f$ be a $\mathcal{C}^{2}$ endomorphism on a connected Riemannian manifold $M$ and let $\Lambda$ be a hyperbolic saddle-type repeller for $f$ such that $f$ is $d$-to- 1 on $\Lambda$, and $f$ has no critical points in $\Lambda$. Consider an arbitrary small $\mathcal{C}^{2}$ perturbation $g$ of $f$ and let $\mu_{g}^{-}$be the inverse SRB measure of $g$ on the respective hyperbolic repeller $\Lambda_{g}$. Then:

a) $e_{g}\left(\mu_{g}^{-}\right) \leq 0$ and $F_{g}\left(\mu_{g}^{-}\right)=\log d$.

b) If $f$ is an Anosov endomorphism on $M$, then there exists a neighbourhood $V$ of $f$ in $\mathcal{C}^{2}(M, M)$ and a set $W \subset V$ such that $W$ is open and dense in the $\mathcal{C}^{2}$ topology in $V$ and s.t for any $g \in W$ we have $e_{g}\left(\mu_{g}^{-}\right)<0$.

Proof. a) If $g$ is close enough to $f$ then it has a repeller $\Lambda_{g}$ close to $\Lambda$, and $g$ is also hyperbolic on $\Lambda_{g}$ and does not have critical points in $\Lambda_{g}$. Since $g$ is a small perturbation of $f$ and since $\Lambda_{g}$ is a repeller, it follows that every point in $\Lambda_{g}$ has exactly the same number $d$ of $g$-preimages in $\Lambda_{g}$. From the discussion above, since $g$ has no critical points, we can construct the inverse SRB measure $\mu_{g}^{-}$which is the equilibrium measure of the stable potential $\Phi_{g}^{s}(x)=\log \left|\operatorname{det} D g_{s}(x)\right|, x \in \Lambda_{g}$; this implies that $\mu_{g}^{-}$is ergodic too, and its Lyapunov exponents are constant $\mu_{g}^{-}$-a.e on $\Lambda_{g}$. Thus from [8], it follows that $\mu_{g}^{-}$is the weak limit of a sequence of measures of type (1), where the degree function $d(\cdot)$ is constant and equal to $d$ everywhere near $\Lambda_{g}$.

This implies then that the Jacobian of $\mu_{g}^{-}$is constant and equal to $d$; indeed for any small borelian set $B$, we have that a point $x \in g(B)$ if and only if there is exactly one $g$-preimage $x_{-1}$ of $x$ in $B$, hence we can use this fact in the above convergence of measures towards $\mu^{-}$. Therefore

$$
F_{g}\left(\mu_{g}^{-}\right)=\int \log J_{g}\left(\mu_{g}^{-}\right)(x) d \mu_{g}^{-}(x)=\log d
$$

And from (2) we have that

$$
h_{\mu_{g}^{-}}(g)=\log d-\sum_{\lambda_{i}\left(\mu_{g}^{-}\right)<0} \lambda_{i}\left(\mu_{g}^{-}\right),
$$

where the negative Lyapunov exponents are repeated according to their respective multiplicities. Thus if $e_{g}\left(\mu_{g}^{-}\right)>0$, it would follow from the $g$-invariance of $\mu_{g}^{-}$, that

$$
F_{g}\left(\mu_{g}^{-}\right)>\int \log |\operatorname{det} D g| d \mu_{g}^{-}=\frac{1}{n} \int \log \left|\operatorname{det} D g^{n}\right| d \mu_{g}^{-}, n \geq 1
$$

Hence from the last two displayed formulas and Birkhoff Ergodic Theorem, we obtain $h_{\mu_{g}^{-}}(g)>$ $\sum_{\lambda_{i}\left(\mu_{g}^{-}\right)>0} \lambda_{i}\left(\mu_{g}^{-}\right)$, which gives a contradiction with Ruelle's inequality. Hence for any perturbation $g$,

$$
e_{g}\left(\mu_{g}^{-}\right) \leq 0
$$

b) From [12] we can construct the SRB measure of $f$, denoted by $\mu_{f}^{+}$, which is the projection by $\pi_{*}$ of the equilibrium measure of $\Phi_{f}^{u}(\hat{x})=-\log \left|\operatorname{det} D f_{u}(\hat{x})\right|, \hat{x} \in \hat{M}$. In particular $\mu_{f}^{+}$is ergodic, hence its Lyapunov exponents are constant $\mu_{f}^{+}$-a.e. If the entropy production $e_{f}\left(\mu_{f}^{+}\right)$were strictly negative, then $F_{f}\left(\mu_{f}^{+}\right)<\int \log |\operatorname{det} D f| d \mu_{f}^{+}$. Since from $[6], h_{\mu_{f}^{+}}(f) \leq F_{f}\left(\mu_{f}^{+}\right)-\sum_{\lambda_{i}\left(\mu_{f}^{+}\right)<0} \lambda_{i}\left(\mu_{f}^{+}\right)$, 
it would follow that $h_{\mu_{f}^{+}}(f)<\sum_{\lambda_{i}\left(\mu_{f}^{+}\right)>0} \lambda_{i}\left(\mu_{f}^{+}\right)$, which is a contradiction to the fact that the SRB measure satisfies Pesin entropy formula. Consequently,

$$
e_{f}\left(\mu_{f}^{+}\right) \geq 0
$$

Now if $e_{f}\left(\mu_{f}^{-}\right)=0$, then $F_{f}\left(\mu_{f}^{-}\right)=\int \log |\operatorname{det} D f| d \mu_{f}^{-}$; hence from the Birkhoff Ergodic Theorem and [6] we obtain:

$$
h_{\mu_{f}^{-}}(f)=\int \log |\operatorname{det} D f| d \mu_{f}^{-}-\sum_{\lambda_{i}\left(\mu_{f}^{-}\right)<0} \lambda_{i}\left(\mu_{f}^{-}\right)=\sum_{\lambda_{i}\left(\mu_{f}^{-}\right)>0} \lambda_{i}\left(\mu_{f}^{-}\right),
$$

where the positive Lyapunov exponents are repeated according to their multiplicities. Thus from the uniqueness of the $f$-invariant measure satisfying Pesin entropy formula, we obtain that $\mu_{f}^{-}=\mu_{f}^{+}$. Recalling from above that $\mu_{f}^{-}$is the equilibrium measure of the stable potential $\Phi^{s}$ and $\mu_{f}^{+}$is the equilibrium measure of the unstable potential $\Phi^{u}$, we see from Livshitz Theorem (see [5]), that $\mu_{f}^{-}=\mu_{f}^{+}$if and only if $|\operatorname{det} D f|$ is cohomologous to a constant.

Now if $g$ is a small perturbation of $f$, it follows from above and from Livshitz Theorem (see for instance [5]), that $|\operatorname{det} D g|$ is cohomologous to a constant if and only if there exists a constant $c$ such that for any $n \geq 1, S_{n}(|\operatorname{det} D g|)(x)=n c, \forall x \in \operatorname{Fix}\left(g^{n}\right)$. As the set of $g$ 's not satisfying the above equalities is open and dense in $V$, we obtain the conclusion.

A particular case where Proposition 1 applies is for a hyperbolic (linear) toral endomorphism $f_{A}$, and for smooth perturbations $g$ of $f_{A}$. Unlike for hyperbolic toral automorphisms, perturbations of hyperbolic toral endomorphisms are not necessarily conjugated to the linear ones (see [11]). For toral endomorphisms $f_{A}$, it is easy to see that the entropy production of any invariant measure is non-positive, i.e $e_{f_{A}}(\mu) \leq 0$. However we see later in Corollary 2 that this is not true for perturbations of $f_{A}$.

Now for an Anosov endomorphism $f$ and an absolutely continuous measure $\rho$ with respect to the Lebesgue measure on $M$, let us consider the measures:

$$
\frac{1}{n} \sum_{0 \leq k<n} \rho \circ f^{-k}
$$

Corollary 1. Consider an Anosov endomorphism $f$ on the compact connected Riemannian manifold $M$ such that $f$ has no critical points in $M$, and $|\operatorname{det} D f|$ is not cohomologous to a constant on $M$. Then the inverse SRB measure $\mu_{f}^{-}$is not a weak limit of a sequence of type (4).

Proof. As was proved in [15], the entropy production of any limit of measures of type (4) is nonnegative. On the other hand, if $|\operatorname{det} D f|$ is not cohomologous to a constant, then $e_{f}\left(\mu_{f}^{-}\right)<0$. Thus in our case $\mu_{f}^{-}$is not a weak limit of measures of type (4).

We now give concrete examples of Anosov endomorphisms and of repellers whose inverse SRB measures have negative entropy production: 
Corollary 2 (Inverse SRB measure with negative entropy production). Consider the hyperbolic toral endomorphism on $\mathbb{T}^{2}$ given by $f(x, y)=(2 x+2 y, 2 x+3 y)(\bmod 1)$ and its smooth perturbation

$$
g(x, y)=(2 x+2 y+\varepsilon \sin 2 \pi y, 2 x+3 y+2 \varepsilon \sin 2 \pi y)(\bmod 1)
$$

Then the inverse SRB measure of $g$ has negative entropy production, while the SRB measure of $g$ has positive entropy production, i.e

$$
e_{g}\left(\mu_{g}^{-}\right)<0 \text { and } e_{g}\left(\mu_{g}^{+}\right)>0
$$

Proof. First of all we notice that $f$ is given by an integer valued matrix $A$ which has one eigenvalue larger than 1 and another eigenvalue in $(0,1)$, hence $f$ is an Anosov endomorphism on $\mathbb{T}^{2}$. Thus for $\varepsilon>0$ small enough, we have that $g$ (which from its expression, is well defined as an endomorphism on $\mathbb{T}^{2}$ ) is hyperbolic as well. We calculate now the determinant of the derivative of $g$ as

$$
\operatorname{det} D g(x, y)=2+4 \pi \varepsilon \cos 2 \pi y
$$

Now, from Proposition 1 we see that $e_{g}\left(\mu_{g}^{-}\right)<0$ if and only if the function $|\operatorname{det} D g|$ is cohomologous to a constant. But this is equivalent from the Livshitz conditions ([5]) to the fact that there exists a constant $c$ such that

$$
S_{n}(|\operatorname{det} D g|)(x)=n c, x \in \operatorname{Fix}\left(g^{n}\right), n \geq 1
$$

In our case let us notice that both $(0,0)$ and $\left(0, \frac{1}{2}\right)$ are fixed points for the map $g$. However

$$
|\operatorname{det} D g(0,0)|=2+4 \pi \varepsilon, \text { whereas }\left|\operatorname{det} D g\left(0, \frac{1}{2}\right)\right|=2-4 \pi \varepsilon
$$

So the Livshitz condition above is not satisfied, and $|\operatorname{det} D g|$ is not cohomologous to a constant. Hence according to Theorem 1 we obtain $e_{g}\left(\mu_{g}^{-}\right)<0$ and $e_{g}\left(\mu_{g}^{+}\right)>0$.

Example. There exist also examples of fractal non-invertible hyperbolic repellers $(f, \Lambda)$ which are not Anosov endomorphisms, but which are $d$-to- 1 for some integer $d$ and we can still construct the inverse SRB measures. We follow here the idea of [8] to employ smooth (say $\mathcal{C}^{2}$ ) perturbations of certain product basic sets. For these examples as well, we can apply the fact that the Jacobian of the inverse SRB measure $\mu^{-}$is constant, the equality in the inverse Pesin entropy formula for the measure $\mu^{-}$(see (2)), and Ruelle's inequality. In this way we obtain the same result as in Proposition 1 a). For instance, let us take $f: \mathbb{P} \mathbb{C}^{1} \times \mathbb{T}^{2} \rightarrow \mathbb{P C}^{1} \times \mathbb{T}^{2}$ given by

$$
f_{g}\left(\left[z_{0}: z_{1}\right],(x, y)\right)=\left(\left[z_{0}^{k}: z_{1}^{k}\right], g(x, y)\right)
$$

where $k \geq 2$ is fixed, and $g$ is a $\mathcal{C}^{2}$ perturbation of a hyperbolic toral endomorphism $f_{A}: \mathbb{T}^{2} \rightarrow \mathbb{T}^{2}$ without critical points. Then $f_{g}$ has a connected hyperbolic repeller $\Lambda:=S^{1} \times \mathbb{T}^{2}$ in the phase space $\mathbb{P}^{1} \mathbb{C} \times \mathbb{T}^{2}$, and $f_{g}$ is $k|\operatorname{det}(A)|$-to- 1 on $\Lambda$. 
Let us consider now the following smooth perturbation of $f_{g}$, namely $f_{\varepsilon, g}: \mathbb{P} \mathbb{C}^{1} \times \mathbb{T}^{2} \rightarrow \mathbb{P} \mathbb{C}^{1} \times \mathbb{T}^{2}$,

$$
f_{\varepsilon, g}\left(\left[z_{0}: z_{1}\right],(x, y)\right):=\left(\left[z_{0}^{k}+\varepsilon z_{1}^{k} \cdot e^{2 \pi i(2 x+y)}: z_{1}^{2}\right], g(x, y)\right)
$$

From its construction we see that $f_{\varepsilon, g}$ is well defined as a $\mathcal{C}^{2}$ endomorphism on $\mathbb{P} \mathbb{C}^{1} \times \mathbb{T}^{2}$. It follows from Proposition 3 of [8] that $f_{\varepsilon, g}$ has a connected hyperbolic repeller

$$
\Lambda_{\varepsilon, g}:=\bigcap_{n \leq 0} f_{\varepsilon, g}^{n}(V)
$$

for a neighbourhood $V$ of $\Lambda$. Also $\Lambda_{\varepsilon, g}$ is close to $\Lambda, f_{\varepsilon, g}$ has stable and unstable directions on $\Lambda_{\varepsilon, g}$, and $f_{\varepsilon, g}$ is $k|\operatorname{det}(A)|$-to- 1 on $\Lambda_{\varepsilon, g}$. The repeller $\Lambda_{\varepsilon, g}$ has a complicated fractal structure with self-intersections. From [8] it follows that the endomorphism $f_{\varepsilon, g}$ has an inverse SRB measure $\mu_{\varepsilon, g}^{-}$ on $\Lambda_{\varepsilon, g}$.

Corollary 3. In the above setting for any $\mathcal{C}^{2}$ perturbation $g$ of a hyperbolic toral endomorphism $f_{A}: \mathbb{T}^{2} \rightarrow \mathbb{T}^{2}$, it follows that the entropy production of the inverse SRB measure of the associated endomorphism $f_{\varepsilon, g}: \mathbb{P} \mathbb{C}^{1} \times \mathbb{T}^{2} \rightarrow \mathbb{P C}^{1} \times \mathbb{T}^{2}$ is negative, i.e

$$
e_{f_{\varepsilon, g}}\left(\mu_{\varepsilon, g}^{-}\right)<0
$$

Proof. Since $\Lambda_{\varepsilon, g}$ is a connected repeller, it follows from Propositions 1 and 3 of [8], that the number of $f_{\varepsilon, g}$-preimages in $\Lambda_{\varepsilon, g}$ of a point from $\Lambda_{\varepsilon, g}$, is constant. Then we can apply [8] (see also (2)) to show that

$$
h_{\mu_{\varepsilon, g}^{-}}\left(f_{\varepsilon, g}\right)=F_{f_{\varepsilon, g}}\left(\mu_{\varepsilon, g}^{-}\right)-\sum_{\lambda_{i}\left(\mu_{\varepsilon, g}^{-}\right)<0} \lambda_{i}\left(\mu_{\varepsilon, g}^{-}\right),
$$

where the Lyapunov exponents are repeated according to their multiplicities. Then as in the proof of Proposition 1 a), we see that $e_{f_{\varepsilon, g}}\left(\mu_{\varepsilon, g}^{-}\right) \leq 0$. However we cannot have $e_{f_{\varepsilon, g}}\left(\mu_{\varepsilon, g}^{-}\right)=0$ since otherwise, as in the proof of Proposition 1 b) it would follow that the inverse SRB measure $\mu_{\varepsilon, g}^{-}$ satisfies the equality in the (usual) Pesin formula. Then from a Volume Lemma (see [12]), this would imply that $\Lambda_{\varepsilon, g}$ is an attractor.

But from construction, the basic set $\Lambda_{\varepsilon, g}$ is a hyperbolic repeller close to $\Lambda$, thus it cannot have a neighbourhood $U$ with $f_{\varepsilon, g}(U) \subset U$; hence $\Lambda_{\varepsilon, g}$ is not an attractor. Therefore for any $\mathcal{C}^{2}$ perturbation $g$ of $f_{A}$, the entropy production of the inverse SRB measure of the associated endomorphism $f_{\varepsilon, g}$ is negative, i.e $e_{f_{\varepsilon, g}}\left(\mu_{\varepsilon, g}^{-}\right)<0$.

\section{References}

[1] R. Bowen, Equilibrium states and the ergodic theory of Anosov diffeomorphisms, Lecture Notes in Mathematics, 470, Springer 1975.

[2] P. Collet and J.P Eckmann, Concepts and Results in Chaotic Dynamics, Springer Verlag, Berlin Heidelberg, 2006. 
[3] R. L. Dobrushin, Ya. G. Sinai, Yu. M. Sukhov, Dynamical Systems of Statistical Mechanics, in Dynamical Systems, Ergodic Theory and Applications, ed. Ya. G. Sinai, vol. 100, Encyclopaedia of Mathematical Sciences, Springer, 2000.

[4] J. P. Eckmann and D. Ruelle, Ergodic theory of strange attractors, Rev. Mod. Physics, 57, 1985, 617-656.

[5] A. Katok and B. Hasselblatt, Introduction to the Modern Theory of Dynamical Systems, Cambridge Univ. Press, London-New York, 1995.

[6] P. D Liu, Invariant measures satisfying an equality relating entropy, folding entropy and negative Lyapunov exponents, Commun. Math. Physics, vol. 284, no. 2, 2008, 391-406.

[7] E. Mihailescu, Unstable directions and fractal dimension for a class of skew products with overlaps in fibers, Math. Zeitschrift 269, 2011, 733-750.

[8] E. Mihailescu, Physical measures for multivalued inverse iterates near hyperbolic repellors, J. Statistical Physics, 139, 2010, 800-819.

[9] E. Mihailescu, Unstable manifolds and Hölder structures associated with noninvertible maps, Discrete and Cont. Dynam. Syst. 14, 3, 2006, 419-446.

[10] W. Parry, Entropy and generators in ergodic theory, W. A Benjamin, New York, 1969.

[11] F. Przytycki, Anosov endomorphisms, Studia Math. 58, 1976, 249-285.

[12] M. Qian, Z. Zhang, Ergodic theory for axiom A endomorphisms, Ergodic Th. and Dynam. Syst., 15, 1995, 161-174.

[13] V. A. Rokhlin, Lectures on the theory of entropy of transformations with invariant measures, Russian Math. Surveys, 22, 1967, 1-54.

[14] D. Ruelle, Smooth dynamics and new theoretical ideas in nonequilibrium statistical mechanics, J. Statistical Physics 95, 1999, 393-468.

[15] D. Ruelle, Positivity of entropy production in nonequilibrium statistical mechanics, J. Statistical Physics 85, 1/2, 1996, 1-23.

[16] D. Ruelle, Elements of differentiable dynamics and bifurcation theory, Academic Press, New York, 1989.

[17] Y. Sinai, Gibbs measures in ergodic theory, Russian Math. Surveys, 27, 1972, 21-69.

[18] B. Solomyak, Measure and dimension for some fractal families, Math. Proceed. Cambridge Phil. Soc., 124 (1998), 531-546.

[19] M. Tsujii, Physical measures for partially hyperbolic surface endomorphisms, Acta Math., 194, 2005, 37-132. 
[20] L. S Young, What are SRB measures and which dynamical systems have them? Dedicated to D. Ruelle and Y. Sinai on the occasion of their 65th birthdays, J. Stat. Phys. 108, 2002, $733-754$.

Eugen Mihailescu, Institute of Mathematics "Simion Stoilow" of the Romanian Academy, P.O. Box 1-764, RO 014700, Bucharest, Romania.

E-mail: Eugen.Mihailescu@imar.ro Webpage: www.imar.ro/ mihailes

Mariusz Urbanski, Department of Mathematics, University of North Texas, P.O. Box 311430, Denton, TX 76203-1430, USA.

Email: urbanski@unt.edu_Webpage : www.math.unt.edu/ urbanski 